\title{
Thalassemia: An Indian Perspective
}

\author{
${ }^{1}$ Ruchika Garg, ${ }^{2}$ Prabhat Agrawal, ${ }^{3}$ Neharika Malhotra, ${ }^{4}$ Narendra Malhotra
}

\begin{abstract}
Thalassemia is a congenital and hereditary disease and comprises a "group" of hematological phenotypes. The most common form of thalassemia is due to a defective beta gene. India has a huge burden of many patients with a $\beta$-thalassemia syndrome and sickle cell disease, but few among them are optimally managed, and allogeneic stem cell transplant is unaffordable for the majority of families. Thalassemia was considered by Caffey to be the best example of skeletal dwarfism and infantilism caused by chronic anemia. Transfusion therapy, which is the mainstay of treatment, allows for normal growth and development and suppresses ineffective erythropoiesis. Hematopoietic stem cell transplantation is the only available treatment with the high cost and scarcity of human leukocyte antigen (HLA)-matched, related donors. Prevention and control of beta-thalassemia disease require the accurate diagnosis of carriers and proper genetic counseling. Prenatal diagnosis can be performed in the first or second trimester of pregnancy by deoxyribonucleic acid (DNA) analysis using polymerase chain reaction (PCR). Since there are 17 mutations as well as rare ones causing beta-thalassemia in Asian Indians, the point mutation detection by reverse dot blot (RDB) allele-specific oligonucleotide hybridization for common mutations along with amplification refractory mutation system (ARMS) techniques has been developed for prenatal diagnosis. The author has tried to explain all the important facts regarding thalassemia in an Indian perspective in this article to help the readers.
\end{abstract}

Keywords: Hemoglobin, Prenatal diagnosis, Thalassemia, Transfusion Therapy.

How to cite this article: Garg R, Agrawal P, Malhotra N, Malhotra N. Thalassemia: An Indian Perspective. World J Anemia 2018;2(1):11-15.

Source of support: Nil

Conflict of interest: None

\section{INTRODUCTION}

Thalassemia is a congenital and hereditary disease and comprises a "group" of hematological phenotypes.

The commonest form of thalassemia is due to a defective beta gene. Homozygous and heterozygous

\footnotetext{
${ }^{1,2}$ Associate Professor, ${ }^{3}$ Consultant, ${ }^{4}$ Managing Director

${ }^{1}$ Department of Obstetrics and Gynecology, Sarojini Naidu Medical College, Agra, Uttar Pradesh, India

${ }^{2}$ Department of Medicine, Sarojini Naidu Medical College, Agra Uttar Pradesh, India

${ }^{3,4}$ Global Rainbow Healthcare, Agra, Uttar Pradesh, India

Corresponding Author: Ruchika Garg, Associate Professor Department of Obstetrics and Gynecology, Sarojini Naidu Medical College, Agra, Uttar Pradesh, India, e-mail: ruchikagarg agra@gmail.com
}

forms exist, the former being thalassemia major and the latter, thalassemia minor. Patients with the homozygous form, who have inherited the trait form both parents, are most severely affected and will have practically no adult hemoglobin $\mathrm{A}$, thereby becoming symptomatic during infancy or early childhood and often dying by adolescence. However, with newer methods of blood transfusion, bone marrow transplantation, and other life-prolonging forms of therapy, many patients are now living to adulthood, are less severely affected, and may initially present during adult life with anemia. The degree of severity of the symptoms and radiological findings in the either group vary widely, and between these two extreme forms lies the clinical entity of thalassemia intermedia. Variations in the alpha chain are clinically unimportant; other variations in the beta chain include sickle cell disease (hemoglobin $S$ ) and hemoglobin C, D, E, and O, all of which are clinically significant. The inheritance of two defective alpha genes (homozygous alpha-thalassemia) is inconsistent with survival, but heterozygous alpha-thalassemia presents with only mild anemia. It is much less common than beta-thalassemia.

Hemoglobin consists of two "alpha-like" chains along with two "beta-like" chains. Hemoglobin molecule is the principal oxygen carrier in the human body. The oxygen requirements of the embryo, fetus, neonate, child, and adults are, however, different. Normal people have diploid cells with tow beta genes (one from each parent), two delta gees, four gamma genes, and two epsilon genes. There are also four alpha genes and two functional zeta genes (only zeta- 2 is functional, zeta-1 is pseudogene).

\section{MOLECULAR EPIDEMIOLOGY}

The question of single or multiple origins for hemoglobin $\mathrm{E}$ in Southeast Asia is still unresolved. ${ }^{1-3}$ Recombination events producing alpha + thalassemia deletion are frequent, whereas alpha 0 thalassemia is produced by a variety of large deletion, each of which has had a single origin. The evidence favoring natural selection by Plasmodium falciparum malaria as the primary tropics and subtropics is getting controversial with conflicting studies. While the mechanism of protection remains unclear, epidemiological evidence supporting the hypothesis is, however, strong, but more information is required from 
case-control studies on the amount of protection provided by the various thalassemia genotypes.

\section{Thalassemia and Malaria}

It is now clear that the thalassemias are the commonest single gene diseases in man, with a widespread distribution throughout the Mediterranean, Middle East, Indian subcontinent, and Southeast Asia. All these ethnic populations have specific types of thalassemia mutations which, presumably, have arisen locally and expanded by selection together with the drift and founder effect. Recent studies have shown that alpha-thalassemia provides protection against severe P. falciparum malaria. ${ }^{4}$

\section{Molecular Pathogenesis}

Beta-thalassemia is clinically more significant in India, with higher incidence in certain communities. ${ }^{5}$ Few common mutations account for $>95 \%$ of severe beta-thalassemia cases. Concurrent presence of alpha-thalassemia modifies the not phenotype of beta-thalassemia in India. Alpha-thalassemia is not a significant problem in India because the severe alpha deletion mutations are less common in this region. The carrier rate for $\beta$-thalassemia varies from 3 to $17 \% .{ }^{6,7}$ The alpha-thalassemia carrier rate in India varies from 1 to $80 \%$ and it is clinically less significant than $\beta$-homozygous thalassemia. Alphathalassemia, if present in $\beta$-homozygous thalassemia patients, ameliorates the phenotype and the majority present as thalassemia intermedia. Hemoglobin E is an abnormal hemoglobin, prevalent in the North Eastern States of India. ${ }^{8-10}$

In India, majority of the patients in the thalassemia major and thalassemia intermedia group have severe beta plus or beta zero mutation. A study from Mumbai showed that six severe and common India mutations [(IVS 1-5 (G->C), 619 bp deletion, IVS 1-1 (G->T), codons 8/9 $(+\mathrm{G})$, codon $15(\mathrm{G}->\mathrm{A})$, codons $41 / 42$ (-CTTT) ] accounted for $92.0 \%$ of molecular lesions in the thalassemia major group, $87 \%$ in the server thalassemia intermedia group, and $73 \%$ in the mild thalassemia intermedia group. ${ }^{9,11}$ IVS $1-1(\mathrm{G}->\mathrm{T})$ and codon $30(\mathrm{G}->\mathrm{C})$ are significantly more common in thalassemia intermedia cases, whereas the mild capsite $+1(\mathrm{~A}->\mathrm{C})$ mutation is present in both severe and mild cases. The other mild beta plus mutations like poly $\mathrm{A}(\mathrm{T}->\mathrm{C}),-28$ $(A->G)$, and $-88(C->T)$ are usually seen only in thalassemia intermedia cases. However, studies performed in a number of India centers have shown poor correlation between the beta genotype and thalassemia phenotype in majority of India patients. ${ }^{12,13}$

\section{Pathophysiology of Anemia in Thalassemia}

Hemolysis and ineffective erythropoiesis cause the anemia that occurs in thalassemia. The relative contributions of these two pathologic processes differ in various forms of thalassemia. The bone marrow of patients with thalassemia contains five to six times the number of erythroid precursors as does the bone marrow of healthy controls, with 15 times the number of apoptotic cells in the polychromatophilic and orthochromic stages. ${ }^{14}$ Accelerated apoptosis, the major cause of ineffective erythropoiesis, is caused by excess $\alpha$-chain deposition in erythroid precursors. Accelerated apoptosis is associated with a rise in extracellular expose of phosphatidylserine, an important signal for removal by activated macrophages, whose numbers are increased in thalasssemic bone marrow. The principal clinical findings in thalassemia relate to those of a microcytic, hypochromic anemia of varying severity. Severe cases of thalassemia major usually manifest themselves during the first year of life ${ }^{15}$ Minor forms of the disease, such as thalassemia minor or the forms of thalassemia combined with other abnormal hemoglobins (such as C, S, H, E, and Lepore), may be asymptomatic for long period, and the diagnosis may only be suggested on routine peripheral blood smear or other hematological work-up.

\section{CLINICAL SPECTRUM OF THALASSEMIA}

Prior to the 1970s, the prognosis of patients with thalassemia was very poor. Death was expected to occur before puberty due to the pathophysiology of the disease. The anemia that is associated with thalassemia may be severe and is accompanied by ineffective erythropoiesis with bone expansion and extramedullary hematopoiesis in the liver, spleen, and other sites, such as paravertebral masses. The head often appears unduly large with a mongoloid facies due to overgrowth of the facial bones secondary to excessive marrow hyperplasia in the maxilla and other paranasal sinuses and facial bones. The combination of sickle cell hemoglobin and beta-thalassemia also produces sickle cell disease of varying severity. ${ }^{16}$ Despite their more benign clinical state, the majority of patients with sickle cell-thalassemia will have skeletal changes, usually related to avascular necrosis and infarction, but without severe marrow hyperplasia.

Hemoglobin E-thalassemia is seen in West Bengal and Assam (where $4 \%$ of the population carries the hemoglobin E gene) and much of Southeast Asia, where the hemoglobin E and thalassemia genes overlap the prognosis is better than in homozygous thalassemia, and most affected individuals reach adulthood, even to 40 or 50 years or most although suffering repeated hemolytic criers. 
Growth and maturation appear to be normal up to 8 to 10 years of age, after which retardation occurs, resulting in short stature premature fusion of the epiphyses of the long bones of the extremities which is a relatively common finding in patients with thalassemia major. There is also a delay in development of the secondary sex characteristics. Thalassemia was considered by Caffey to be the best example skeletal dwarfism and infantilism caused by chronic anemia.

\section{THERAPY}

Transfusion therapy, which is the mainstay treatment, allows for normal growth and development and suppresses ineffective erythropoiesis. Regular transfusion therapy to maintain hemoglobin levels of at least 9 to 10 $\mathrm{gm} / \mathrm{dL}$ allows for improved growth and development and also reduces hepatosplenomegaly due to extramedullary hematopoiesis as well as bone deformities. Although initiation of blood transfusion therapy significantly increases life expectancy by controlling anemia of iron in the tissues, posing significant risk of severe cardiac, endocrinological, and hepatic complication, which are fatal if not prevented. Thus, iron overload, either through increased iron adsorption caused by erythropoietic activity or as a result of transfusional iron loading, constitutes the most important complication in $\beta$-thalassemia and is the major focus for clinical management. ${ }^{17}$

\section{Transfusion-transmitted Infections}

Primarily hepatitis B and hepatitis C are an important cause of death in countries where proper testing is not available. Importance of proper donor screening and testing of blood for transfusion-transmitted infections cannot be overemphasized. ${ }^{12}$ The endocrinopathies can now be treated with hormone replacement.

However, the most serious result of iron overload is life-threatening cardiotoxicity, for which chelation therapy is required. In the past 20 years, the treatment of thalassemia major has vastly improved in countries with access to adequate and safe blood and iron chelation therapy with desferrioxamine. Experimental therapies to ameliorate the anemia include fetal hemoglobin modifiers (5-Aza, hydroxyurea, hydroxybutyrates) antioxidant (plant flavonoids, polyphenols). ${ }^{18}$

The combination of blood transfusion and iron chelation is now the reference treatment for thalassemia major. ${ }^{19}$ Blood transfusion is necessary to maintain for thalassemia with a level of hemoglobin high enough to supply an adequate supply of oxygen to the tissues and to suppress the production of (defective) red cells. ${ }^{20}$ Super transfusion regimens evolved between 1979 and
1981, shifting the goal from simply correcting symptomatic anemia to systematically maintaining a minimum hemoglobin level above $12 \mathrm{gm} / \mathrm{dL}$. While beneficial in combating anemia, super transfusion regimens increased the degree of iron overload and its consequences. ${ }^{21}$ Therefore, current recommendations favor transfusion regimens maintaining a pretransfusion hemoglobin level of 9 to $10.5 \mathrm{gm} / \mathrm{dL}$. Leukodepletion filters and irradiated red cells are the ideal products for transfusion. In addition, the early detection of iron-related complication is actively pursued, and their aggressive treatment has become standard practice.

Because of the repeated transfusions, iron overload occurs after a number of years (the time varies). There is no simple relationship between iron overload and serum iron or serum ferritin concentration. The degree of iron accumulation can be determined on a liver biopsy. The risk of severe endocrine, liver and heart complications increases considerably with iron concentrations of more than $15 \mathrm{mg} / \mathrm{gm}$ dry weight of liver. ${ }^{22}$ Iron chelation treatment should be started when serum levels reach about 1,000 $\mu \mathrm{g} / \mathrm{L}$, which usually occurs after the first 10 to 20 transfusions (near 3 years of age). Desferrioxamine is infused via a thin needle inserted subcutaneously and connected by an infusion line to a portable pump. The infusion continues over 8 to 10 hours and is given 5 to 7 times per week at a mean daily dose of 20 to $50 \mathrm{mg} / \mathrm{kg}$ body weight. Urinary iron excretion of $0.5 \mathrm{mg} / \mathrm{kg} /$ day is generally indicative of negative iron balance. This is a difficult treatment and requires strict compliance. Usually, 250 to $500 \mathrm{mg}$ vitamin $\mathrm{C}$ is given simultaneously because this increases the effectiveness (iron excreted in urine). Attempts are made to keep the iron content in the liver at less than 3 to $7 \mathrm{mg} / \mathrm{gm}$ dry liver. During treatment, the urine is colored brownish orange by the iron. The dose of desferrioxamine should be adjusted according to body iron stores and age. Dose adjustment can be made with reference to the serum ferritin level using therapeutic index:

\section{THERAPEUTIC INDEX}

$$
\text { Therapeutic index }=\frac{\text { mean daily dose }(\mathrm{mg} / \mathrm{kg})}{\text { ferritin }(\mu \mathrm{g} / \mathrm{L})}
$$

The aim is to keep the index $<0.025$ at all times. Using this index will assist in reducing the risk of toxicity associated with excess chelation. The probability of survival, free from cardiac disease has been shown to increase to $91 \%$, provided that at least two-thirds of serum ferritin values remain below $2.500 \mu \mathrm{g} / \mathrm{L}$. Recently, hepatic iron levels were shown to correlate well the risk of clinical disease. Maintenance of hepatic iron concentration below $15 \mathrm{mg} /$ gm (dry weight) has been associated with decreased risk of severe cardiac disease and death. Deferiprone (L1), an 
orally administered chelator, is safe and effective. Longterm administration of deferiprone does not appear to be associated with liver damage as was reported earlier. Adverse effects of deferiprone include arthralgia, nausea, and other gastrointestinal symptoms, fluctuating liver enzyme levels, leukopenia, and rarely agranulocytosis and zinc deficiency. Most of these effects can be monitored and controlled. It has a number of advantages over deferoxamine.

Furthermore, the compliance of patients is improved with the use of combined therapy because fewer painful injections of deferoxamine are required. The newly approved oral iron chelator deferasirox (ICL670) is particularly promising for its efficacy, which may be similar to that of deferoxamine. Deferasirox (Exjade) is administered once daily and appears to have an acceptable side effect profile.

\section{Hematopoietic Stem-cell Transplantation}

Hematopoietic stem-cell transplantation is the only available treatment with the high cost and scarcity of HLAmatched, related donors. The past several years have brought selection, and the development or alternative sources of hematopoietic stem cell. Low-risk patients, those with thalassemia termed as class I or class II by the Lucarelli classification (degree of hepatomegaly, presence of portal fibrosis on liver biopsy, and the effectiveness of chelation therapy before transplantation) have excellent results after bone marrow transplantation; but patients with class III disease (with extensive liver damage from iron overload) have poor results.

An increase in the available donor pool for bone marrow transplantation has been achieved by using matched, unrelated donors. ${ }^{12,23}$ Extended haplotyping has been developed for unrelated donors. The use of related or unrelated umbilical-cord blood has further increased the donor pool. Hematopoietic stem-cell transplantation with the use of related or unrelated donor is a viable alternative that generally results in excellent outcome for low-risk patients. If transplantation is successful, transfusions, and usually chelation therapy, are no longer necessary and becomes the most cost-effective modality of management. ${ }^{23-25}$

\section{Gene Therapy}

The therapeutic strategy involves the insertion of a normally functioning gamma-globin or beta-globin gene into the patient's autologous hematopoietic stem cells. ${ }^{26}$ Although the concept appears relatively simple, it has met with two decades of seemingly insurmountable hurdles in introducing the desired gene through appropriate vector construction. These types of therapies remain highly experimental; accordingly, their clinical potential remains uncertain. Nevertheless, these methods may be useful molecular approaches for the development of new therapies for thalassemias in future. ${ }^{27-31}$

\section{Prenatal Diagnosis}

Polymerase chain reaction technology has been used for more than a decade to delete point mutation or deletions in chorionic villus samples, enabling first-trimester, DNAbased testing for thalassemia. Thalassemia is the most common single gene disorder and is widely distributed in Asia. Indians have an average prevalence rate of $4 \%$, with a high prevalence among Sindhis, Punjabis, Gujratis, and Bengalis. Prevention and control of beta-thalassemia disease require the accurate diagnosis of carriers and proper genetic counseling. Prenatal diagnosis can be performed in the first or second trimester of pregnancy by DNA analysis using PCR. Since there are 17 mutations as well as rare ones causing beta-thalassemia in Asian Indians, the point mutation detection by RDB allele-specific oligonucleotide hybridization for common mutations along with ARMS techniques have been developed for prenatal diagnosis. Maternal contamination of fetal DNA is normally ruled out by the variable number of tandem repeat analysis using apolipoprotein B site. Patients are counseled to continue the pregnancy when the fetuses are either normal or have traits, whereas in the case of an affected fetus, the patients are advised termination of the pregnancy. Prenatal diagnosis of beta-thalassemia by the RDB or ARMS technique can prevent the birth of an affected child in developing countries in which betathalassemia is quite prevalent.

Before implantation, genetic diagnosis is now feasible and involves performing conventional in vitro fertilization, followed by removal of one or two cells from the resulting blastomeres on day $3 .^{32}$ The PCR method is then used to detect thalassemia mutations within the cells that have been removed, so that unaffected blastomeres may be selected for implantation. Prior to implantation, genetic diagnosis is extended to HLA typing on embryonic biopsies, which allows the selection of an embryo that is not affected by thalassemia and that may also serve as a stem-cell donor for a previously affected child within the same family.

\section{REFERENCES}

1. Nadkarni A, Gorakshakar AC, Lu CY, Krishnamoorthy R, Ghosh K, Colah R, Mohanty D. Molecular pathogenesis and clinical variability of beta-thalassaemia syndromes among Indians. Am J Heamatol 2001 Oct;68(2):75-80.

2. Panigrahil,AgarwalS,Pradhan M,Choudhry DR, Choudhry VP, Saxena R. Molecular characterization of thalassaemia intermedia in Indians. Haematologica 2006 Sep;91:1279-1280. 
3. Bandyopadhyay S, Roychowdhury K, Chandra S, Das M, Dasgupta UB. Variable severity of beta-thalassaemia and $\mathrm{xmnl}$ polymorphism. Clin Exp Med 2001 Sep;1(3):155-159.

4. Panigrahi I, Marwaha RK. Mutational spectrum of thalassaemia in India. Indian J Hum Genet 2007 Jan-Apr;13(1):36-37.

5. Kulozik AE. $\beta$-Thalassaemia: molecular pathogenesis and clinical variability. Eur J Paediatr 2007;151(5):78-84.

6. Efremov GD. Dominantly inherited beta-thalassaemia. Hemoglobin 2007;31(2):193-207.

7. Borgna-Pignatti C. Modern treatment of thalassaemia intermedia. Br J Haematol 2007 Aug;138(3):291-304.

8. Hill AV. Molecular epidemiology of the thalassaemia (including haemoglobin E). Baillieres Clin Haematol 1992 Jan;5(1):209-238.

9. Old JM, Khan SN, Verma I, Fucharoen S, Kleanthus M, Ioannou P, Kotea N, Fisher C, Riazuddin S, Saxena R, et al. A multi-center study in order to further define the molecular basis of beta-thalassaemia in Thailand, Pakistan, Sri Lanka, Mauritius, Syria, and India to develop a simple molecular diagnostic strategy by amplification refractory mutation system-polymerase chain reaction. Hemoglobin 2001 Nov;25(4):397-407.

10. Sen R, Chakrabarti S, Sengupta B, De M, Haldar A, Poddar S, Gajra B, Talukder G. Alpha-thalassaemia among tribal populations of Eastern India. Hemoglobin 2005;29(4):277-280.

11. Ward A, Caro JJ, Green TC, Huybrechts K, Arana A, Wait S, Eleftheriou A. An international survey of patients with thalassaemia major and their views about sustaining life-long desferrioxamine use. BMC Clin Pharmacol 2002 Apr;2:3.

12. Kanga U, Panigrahi A, Kumar S, Mehra NK. Asian Indian donor marrow registry: All India Institute of Medical Sciences experience. Transplant Proc 2007 Apr;39(3):719-720.

13. Colah R, Mohanty D. Beta-thalassaemia: expression, molecular mechanisms and mutations in Indians. India J Pediatr 1999 Jan-Feb;66(1):36.

14. Karimi M, Ghiam AF, Hashemi A, Alinejad S, Soweid M, Kashef S. Bone mineral density in beta-thalassaemia major and intermedia. Indian Pediatr 2007 Jan;44(1):29-32.

15. Saini A, Chandra J, Goswami U, Singh V, Dutta AK. Case control study of psychosocial morbidity in beta thalassaemia major. J Pediatr 2007 May;150(5):516-520.

16. Balgir RS. Control and prevention of the genetic load of haemoglobinopathies in India. Natl Med J India 1999 SepOct;12(5):234-238.

17. Bradai M, Pissard S, Abad MT, Dechartres A, Ribeil JA, Landais $\mathrm{P}$, de Montalembert M. Decreased transfusion needs associated with hydroxyurea therapy in Algerian patients with thalassaemia major or intermedia. Transfusion 2007 Oct;47(10):1830-1836.

18. Shaji RV, Eunice SE, Baidya S, Srivastava A, Chandy M. Determination of the breakpoint and molecular diagnosis of a common alpha-thalassamia-1 deletion in the Indian population. Br J Haematol 2003 Dec;123(5):942-947.

19. George A, Bhaduri A, Choudhary VP. Development of secondary sex characteristics in multitranfused thalassaemia children. Indian J Pediatr 1997;64(6):855-859.

20. Shaji RV,EdisonES, Krishnamoorthy R,Chandy M,Srivastava A. $\mathrm{Hb}$ Lepore in the Indian population. Hemoglobin 2003 Feb;27(1):7-14.

21. Sankar VH, Arya V, Tewari D, Gupta UR, Pradhan M, Agarwal S. Genotyping of alpha-thalassaemia in microcytic hypochromic anemia patients from North India. J Appl Genet 2006;47(4):391-395.

22. Aesspos A, Kati M, Farmakis D. Heart disease in thalassaemia intermedia: a review of the underlying pathophysiology. Haematologica 2007 May;92(5):658-665.

23. Williams TN, Maitland K, BennettS, Ganczakowski M, Peto TE, Newblod CL, Bowden DK, Weatherall DJ. High incidence of malaria in alpha-thalassaemia children. Nature 1994 Oct;383(6600):480-481.

24. Colah R, Nadhkarni A, Gorakshakar A, Phanasgaonkar S, Surve R, Subramaniam PG, Bondge N, Pujari K, Ghosh K, Mohanty D. Impact of beta globin gene mutations on the clinical phenotype of beta thalassaemia in India. Blood Cells Mol Dis 2004 Sep-Oct;33(2):153-157.

25. Kumar L. Haematopoietic stem cell transplantation: current status. Natl Med J India 2007 May-Jun;20(3):128-137.

26. Bandyopadhyay A, Bandyopadhyay S, Chowdhury MD, Dasgupta UB. Major beta-globin gene mutations in eastern India and their associated haplotypes. Hum Hered 1999 Jul;49(4):232-235.

27. Bashyam MD, Bashyam L,SavithriGR, Gopikrishna M,Sangal V, Devi AR. Molecular genetic analyses of beta-thalassaemia in South India reveals rare mutations in the beta-globin gene. J Hum Genet 2004;49(8):408-413.

28. Agarwal S, Gupta UR, Sarwai S, Phadke S, Agarwal SS. Prenatal diagnosis in beta-thalassaemia: an India experience. Fetal Diagn Ther 2003 Sep-Oct;18(5):328-332.

29. Bandyopadhyay A, Bandyopadhyay S, Basak J, Mondal BC, Sarkar AA, MajumdarS, Das MK, Chandra S, Mukhopadhyay A, Sanghamita M, et al. Profile of beta-thalassaemia in eastern India and its prenatal diagnosis. Prenat Diagn 2004 Dec;24(12): 992-996.

30. Nadkarni A, Sakaguchi T, Gorakshakar A, Phanasgaonkar S, Colah R, Mohanty D, Kiyama R. Three novel polymorphisms in the beta globin gene. Am J Hematol 2005 Oct;80(2): 161-163.

31. Panigrahi I, Marwaha RK. Common queries in thalassaemia care. Indian Pediatr 2006 Jun;43(6):513-518.

32. Verma IC, Bijarnia S. The burden of genetic disorders in India and a framework for community control. Community Genet 2002;5(3):192-196. 